\title{
44586 - NECK AUSCULTATION AS A MEANS OF CONFIRMING ENDOTRACHEAL INTUBATION - A PILOT STUDY
}

\section{Jenifer Ballen, University of Manitoba, Winnipeg, MB, Canada; Christopher Christodoulou, St Boniface General Hospital;}

INTRODUCTION: Neck auscultation is a novel means of confirming endotracheal tube (ETT) intubation. This technique, used during laryngoscopy, involves auscultation of the lateral neck, at the level of the cricoid cartilage, for a characteristic rub made by the ETT as it contacts tracheal rings. This distinctive sound, amplified by an electronic stethoscope, presumably is absent in the event of esophageal intubation. This method, utilized prior to patient ventilation avoids the possible complication of gastric insufflation and aspiration. This pilot study was undertaken to determine if auscultation of the lateral neck during ETT had the same reliability as end tidal carbon dioxide capnography (EtCO2cap) in the detection of esophageal and tracheal intubation.

METHODS: Upon institutional ethics approval, 221 patients undergoing general anesthesia at a tertiary Canadian hospital were recruited for this prospective validation study. The auscultator, blinded during intubation, listened over the lateral aspect of the neck, at the level of the cricoid cartilage. The 3M Littmann Electronic Stethoscope Model 4000 was used during auscultation. The initial intubation maneuver, or first pass of the ETT through either the glottis and trachea or the esophagus was referred to as the first pass maneuver (FPM). After ensuring proper ETT depth, the intubator gently advanced and withdrew the ETT no more than $0.5 \mathrm{~cm}$, for 3 seconds in a technique referred to as the advance and withdrawal maneuver (AWM). The auscultator immediately left the operating theatre and recorded the presence of tracheal or esophageal intubation during FPM and AWM without knowledge of EtCO2cap. Meanwhile, the intubator proceeded with the case and recorded presence or absence of EtCO2cap.

RESULTS: Six esophageal intubations were detected by absent end tidal capnography during this investigation. Two of the esophageal intubations were detected by AWM, and all 213 tracheal intubations were detected by AWM yielding a sensitivity and specificity of $33.3 \%$ and $100 \%$, respectively. A one-tail p value of 0.062 was computed using the Exact version of McNemer's test for correlated proportions. The FPM detected esophageal intubation in all six cases of esophageal intubation for a sensitivity of $100 \%$. However, the FPM detected esophageal intubation in 138 tracheal intubations for a specificity of $35.2 \%$. A p value of $<0.001$ was computed using McNemer's test.

DISCUSSION: This is the first published report of the novel use of an electronic amplifying stethoscope as a means of confirming ETT placement. In this validation study, we were able to use this technique quickly and easily. However, we were unsuccessful in our attempt to confirm our hypothesis that the reliability in detection of esophageal intubations was as good with auscultation of the lateral neck as it is with EtCO2cap. 